\title{
An investigation of the effect of tissue ischaemia upon DNA damage protein markers in ovarian cancer
}

\author{
Sara Tomassini ${ }^{1}$, Lucy Gentles ${ }^{1}$, Calum Kirk ${ }^{1}$, Paul Cross ${ }^{2}$, Huw Thomas ${ }^{1}$, Steve Wedge ${ }^{1}$, Nicola Curtin ${ }^{1}$, \\ Rachel O’Donnell ${ }^{1}$ \\ ${ }^{1}$ Newcastle University, UK \\ ${ }^{2}$ Pathology Centre, Queen Elizabeth Hospital, Gateshead Health NHS Foundation Trust, UK
}

Background: Hypoxia is observed in many aggressive solid organ malignancies and has been associated with poor survival and resistance to chemoradiotherapy. Hypoxia-inducible factor 1 alpha (HIF-1 $\alpha$ ), which is expressed in hypoxic tissues, is detected in most ovarian cancer but alone does not predict response. Expression of DNA repair proteins are also under investigation as potential predictive biomarkers. It is crucial to determine the extent to which intra-operative and postoperative sample collection methods impact on ischemia, and hence HIF-1 $\alpha$, and other biomarkers for successful stratification of targeted therapies.

Method: Tumour samples from patients undergoing surgery for ovarian cancer were harvested and sections were fixed in formalin or snap frozen immediately and after 30,60 and 120 minutes. Nude mice bearing human tumour xenografts were culled and the tumours excised and snap frozen at the same time intervals. Frozen samples were pulverised and homogenised with Phosphosafe (Millipore Sigma) and protease inhibitors. Protein expression was analysed by Western Blotting. Protein expression in a TMA of the FFPE samples was determined by IHC.

Results: Xenograft samples demonstrate a time-dependent increase in HIF1- $\alpha$ at 60 minutes with no changes in DNA repair proteins levels (PARP1, RAD51, DNAPKcs, phosphorylated DNAPKcs) by Western blot. Despite standardisation of sample collection intra-operatively there was significant variability in baseline expression of HIF1- $\alpha$ in patient samples as well as changes in expression with ischaemic time. Expression of DNA repair proteins showed highly variable time-related changes.

Conclusion: Variable pre-analytical sample handling may result in substantial effects upon tissue hypoxia and DNA repair biomarker expression. Pre-existing patient factors and overall differences within the tumour specimens collected intra-operatively, as well as sample collection and processing, may contribute to the highly variable results observed in this pilot study. Studies are ongoing to optimise sample collection and improve reproducibility and standardisation of future biomarker studies. 